\title{
Uma proposta de framework para simulação de casas inteligentes usando Unity
}

\author{
Cael G. B. Santos ${ }^{1}$, Gabriela D. S. Sousa ${ }^{1}$, Josivan R. Reis ${ }^{2}$, Roberto P. Nascimento ${ }^{1}$ \\ ${ }^{1}$ Instituto de Engenharia e Geociências - Universidade Federal do Oeste do Pará \\ (UFOPA) Santarém - PA - Brasil \\ ${ }^{2}$ Universidade Federal do Oeste do Pará (UFOPA) - Oriximiná - PA - Brasil \\ \{roberto.nascimento, josivan.reis\}@ufopa.edu.br \\ \{cael.santos, gabriela.dss\}@discente.ufopa.edu.br
}

\begin{abstract}
With the growing development of new technologies in the field of domotics and Internet of Things new opportunities for the creation of smart environments for users that lack some kind of assistive technology arises. Taking into account the costs associated with the construction of this kind of environment, simulators become an important component for it's development as a way to minimize the time and cost of the project's implementation. Thus, this project presents a proposal of a framework to simulate smart homes that meets the necessities and specifities of the user.
\end{abstract}

Resumo. Com o crescente desenvolvimento de novas tecnologias no campo da domótica e Internet das Coisas surgem novas oportunidades para a criação de ambientes inteligentes para usuários que carecem de algum tipo de tecnologia assistiva. Considerando os custos associados à construção desse tipo de ambientes, simuladores se tornam um componente importante para o seu desenvolvimento, de modo a minimizar o tempo e o custo de implementação do projeto. Assim, este trabalho apresenta uma proposta de framework para simular casas inteligentes que atendam as necessidades e especificidades do usuário.

\section{Introdução}

Segundo o [IBGE 2008], na projeção da população brasileira para as próximas décadas é observado um significativo aumento no número de idosos, ou seja, da população com 65 anos ou mais. Somado a isso, o censo do IBGE de 2010 [IBGE 2010] mostra que mais de 4 milhões de pessoas possuem deficiência motora desde as mais leves até as mais graves. Diante disso, percebe-se a necessidade de minimizar os desafios encontrados frequentemente pela população pertencente a esses grupos. Ademais, com o crescente desenvolvimento na área de automação residencial e IoT (Internet das Coisas), novas oportunidades para a criação de tecnologias assistivas surgiram, no entanto, o custo e o tempo necessário para implementação dessas tecnologias em um ambiente real é um fator limitante para esse tipo de pesquisa. Assim, simulações digitais são desenvolvidas com o intuito de testar diferentes configurações de sensores e criar dados de treino para inteligências artificiais.

O trabalho de [Rodrigues et al. 2020] ressalta que com o envelhecimento populacional, ações que busquem assegurar o conforto e qualidade de vida dos idosos dentro do 
âmbito habitacional, são merecedoras de destaque. Isso em vista que a moradia é uma das dimensões a ser considerada para a garantia da qualidade de vida, principalmente para os idosos e pessoas com limitação motora, os quais passam um maior período de tempo dentro das residências do que os outros grupos etários. Portanto, o planejamento adequado das residência é de fundamental importância para a existência de um espaço com acessibilidade e funcionalidades adequadas as suas necessidades.

Em [Synnott et al. 2015] é apresentado duas formas de se aproximar a criação de uma simulação de casa inteligente: (i) Model based Approaches, com a criação de modelos que definem a ordem, duração e probabilidade da ocorrência de uma ação por um agente simulado, e (ii) Interactive Approaches que são modelos em que o usuário tem controle total ou parcial do agente de simulação.

Com isso, é proposto um framework para simulação de casas inteligentes, baseada no Model based Approaches, utilizando a plataforma Unity ${ }^{\circledR}{ }^{1}$. O framework tem como objetivo facilitar o desenvolvimento de simulações de casas-inteligentes para criar soluções de automação que se adéquem às necessidades específicas do usuário com algum tipo de limitação motora ou cognitiva. Com os agentes e sensores configuráveis é possível simular uma variedade de elementos de IoT e verificar a interação da rotina do usuário com o ambiente a qual está inserido e, assim, gerar dados para treinar sistemas inteligentes voltados para pessoas que carecem de tecnologias assistivas.

\section{Desenvolvimento do Modelo de Simulação com Unity}

A construção do modelo de simulação da casa inteligente foi dividida em dois grupos: Agente e Sensores. O grupo Agente diz respeito as ferramentas que definem o comportamento e a forma com que o agente simulado interage com o ambiente, enquanto o grupo Sensores busca simular os principais sensores utilizados no mercado. Para o desenvolvimento destes teve-se como foco possibilitar criar simulações para diferentes cenários de forma fácil e prática.

No editor do Unity® tem-se o agente da simulação e os objetos que compõe o ambiente da simulação, como demonstrado na Figura 1. O agente possui um módulo responsável por interpretar a rotina do usuário (Fixed Routines) e um módulo responsável por interpretar as instruções de uma atividade (XML Activities Interpreter). Todos os objetos presentes na cena, incluindo os sensores, estão listados no campo a esquerda. Todos os sensores na simulação escrevem seu estado para um arquivo de saída do tipo csv, chamada de SensorOutput.csv, conforme a Figura 2.

\subsection{Agente}

O agente da simulação é a interação do usuário com o ambiente simulado. A rotina do usuário é definida usando um arquivo XML contendo a atividade e o momento a ser realizada. Nesse arquivo, uma atividade é definida usando passos menores que juntos formam a atividade. Cada passo corresponde a uma ação básica da rotina do usuário, como: ir à algum lugar ou esperar durante um certo período de tempo. O Unity ${ }^{\circledR}$ possui um sistema para executar certas funções denominado co-rotinas (Coroutines), esse sistema permite que essas funções possam ser interrompidas e retomadas do ponto em que pararam du-

\footnotetext{
${ }^{1}$ https://unity.com
} 


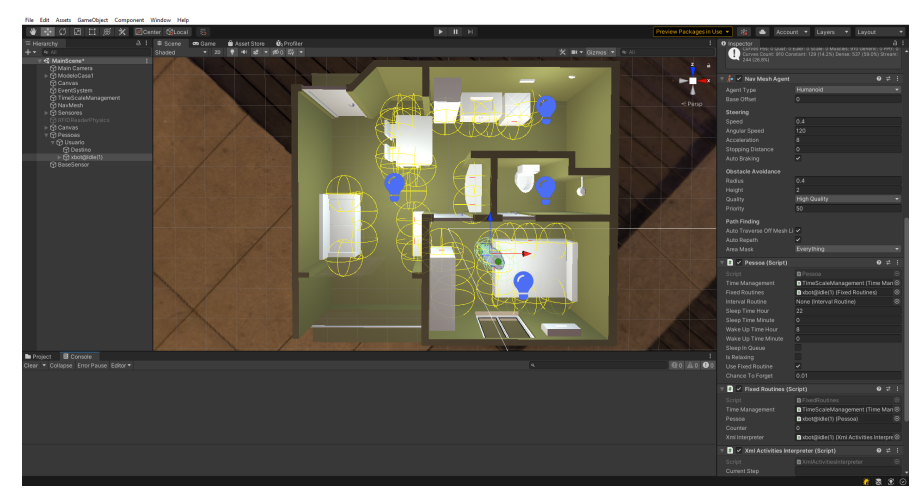

Figura 1. Visão geral do ambiente de simulação no editor do Unity®

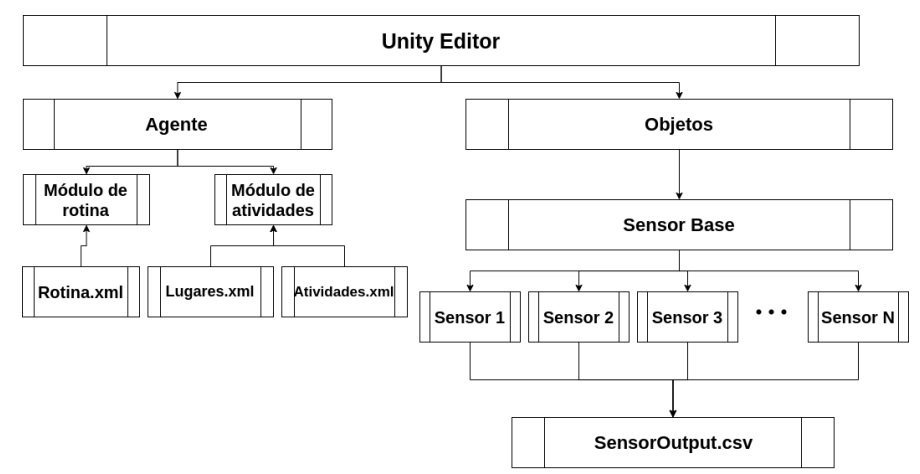

Figura 2. Visão geral do framework de simulação da casa inteligente

rante a execução do programa. Com isso, é possível interromper ou atrasar a execução de certas tarefas a depender da sua prioridade.

\subsection{Sensores}

Os sensores consistem em um conjunto de classes que podem ser criadas como um objeto independente dentro do editor do Unity ${ }^{\circledR}$ ou como uma propriedade que pode ser designada à um objeto já existente. Todas as classes pertencentes ao grupo dos sensores escrevem o seu estado em um arquivo, do tipo CSV, em um intervalo de tempo definido para que esses dados possam ser tratados.

Na simulação pode ser utilizado um gama de sensores diferentes, como: Sensor de movimento que detecta se um objeto entrou na área de cobertura do sensor; sensor RFID que detecta se determinado objeto - neste caso um leitor RFID - está dentro do alcance do sensor; sensor de vibração que detecta se há colisão entre objetos; sensor de pressão que detecta se o agente passou pelo sensor, entre outros. Todos os sensores utilizam o sistema de colisão do Unity®.

\section{Resultado}

Como resultado preliminar, o ambiente de simulação foi implementado e, com ele, é possível criar atividades, simular a rotina diária do usuário em uma casa inteligente, gerar e guardar os dados dos sensores que podem ser transferidos para programas externos para tratamento. Os dados gerados podem ser usados para testar ou treinar algoritmos para implementação de projetos práticos. O uso do Unity permite que modelos e animações 
possam ser criados em programas de terceiros e facilmente importados para o ambiente da simulação, o que torna a modelagem de diferentes cenários mais flexível e menos dependente da capacidade do simulador. Isso é especialmente verdade quando consideramos a grande quantidade de extensões disponíveis para o Unity. Também foi possível observar que ocorre pouca variância nas atividades ao longo de múltiplos dias.

\section{Considerações Finais}

Este trabalho propôs um framework para simular casas inteligentes, permitindo projetar ambientes que atendam as necessidades e especificidades de pessoas que carecem de domótica como tecnologia assistiva. Com isso, o sistema permite definir atividades e rotinas do usuário de forma simples, criar sistema de sensores, simular uma variedade de dispositivos IoT e analisar os seus impactos em um ambiente de casa inteligente.

Embora o sistema para definir atividades e rotinas seja útil para simular situações específicas, a precisão e qualidade da simulação depende da precisão das rotinas e atividades descritas pelo usuário. Por causa disso, modelos que geram uma rotina diária baseado nas necessidades do usuário como os propostos por [Lee et al. 2016] e [Renoux and Klügl 2018] surgem como uma alternativa e será testado no decorrer do desenvolvimento deste projeto. Como trabalho futuro, busca-se finalizar o desenvolvimento do sistema de sensores e adaptar o código-fonte ${ }^{2}$ para que o projeto possa ser distribuído pela Unity Asset Store ${ }^{3}$ e integrado a projetos já existentes.

\section{Agradecimentos}

O presente projeto foi desenvolvido com apoio da Fundação Amazônia de Amparo a Estudos e Pesquisas - FAPESPA através do auxílio a projeto de pesquisa, $\mathrm{n}^{\circ}$ do processo 2019/585931, termo de outorga $n^{\circ} 006 / 2020$.

\section{Referências}

IBGE (2008). Projeção da população do brasil e das unidades da federação. Disponível em: https://www.ibge.gov.br/apps/populacao/projecao/index. html. Acessado em 01 de Abril de 2021.

IBGE (2010). Características Gerais da População, Religião e Pessoas Com Deficiência. Censo Demográfico 2010, pages 1-215.

Lee, W., Cho, S., Chu, P., Vu, H., Helal, S., Song, W., Jeong, Y. S., and Cho, K. (2016). Automatic agent generation for iot-based smart house simulator. Neurocomputing.

Renoux, J. and Klügl, F. (2018). Simulating daily activities in a smarthome for data generation. Proceedings of the 2018 Winter Simulation Conference.

Rodrigues, J. L. d. S., Monteiro, A. G. d. S., Lima, E. K. A., and Silva, G. B. (2020). Analysis of housing in the axes of accessibility and functionality of a housing unit designed for the elderly. Research, Society and Development.

Synnott, J., Nugent, C., and Jeffers, P. (2015). Simulation of smart home activity datasets. Sensors (Switzerland).

\footnotetext{
${ }^{2}$ https://github.com/B-Cut/Unity-SmartHome-Simulator

${ }^{3}$ https://assetstore.unity.com
} 